University of Nebraska - Lincoln

DigitalCommons@University of Nebraska - Lincoln

Faculty Publications in Computer \& Electronics Electrical \& Computer Engineering, Department Engineering (to 2015)

$1-2011$

\title{
A Novel Adaptive Distributed Cooperative Relaying MAC Protocol for Vehicular Networks
}

Ting Zhou

University of Nebraska-Lincoln, tzhou@unlnotes.unl.edu

Hamid Sharif

University of Nebraska-Lincoln, hsharif@unl.edu

Michael Hempel

University of Nebraska-Lincoln, mhempel2@unl.edu

Puttipong Mahasukhon

University of Nebraska-Lincoln, pmahasukhon@huskers.unl.edu

Wei Wang

University of Nebraska-Lincoln, wwang@unlnotes.unl.edu

See next page for additional authors

Follow this and additional works at: https://digitalcommons.unl.edu/computerelectronicfacpub

Part of the Computer Engineering Commons

Zhou, Ting; Sharif, Hamid; Hempel, Michael; Mahasukhon, Puttipong; Wang, Wei; and Ma, Tao, "A Novel Adaptive Distributed Cooperative Relaying MAC Protocol for Vehicular Networks" (2011). Faculty Publications in Computer \& Electronics Engineering (to 2015). 92.

https://digitalcommons.unl.edu/computerelectronicfacpub/92

This Article is brought to you for free and open access by the Electrical \& Computer Engineering, Department of at DigitalCommons@University of Nebraska - Lincoln. It has been accepted for inclusion in Faculty Publications in Computer \& Electronics Engineering (to 2015) by an authorized administrator of DigitalCommons@University of Nebraska - Lincoln. 


\section{Authors}

Ting Zhou, Hamid Sharif, Michael Hempel, Puttipong Mahasukhon, Wei Wang, and Tao Ma 


\title{
A Novel Adaptive Distributed Cooperative Relaying MAC Protocol for Vehicular Networks
}

\author{
Ting Zhou, Hamid Sharif, Michael Hempel, Puttipong Mahasukhon, Wei Wang, Tao Ma
}

\begin{abstract}
Explosive growth in Information Technology has enabled many innovative application areas such as large-scale outdoor vehicular networks for vehicle-to-vehicle communications. By providing time-sensitive and location-aware information, vehicular networks can contribute to a safer and more efficient driving experience. However, the performance of vehicular networks requires robust and real-time data communications and is impacted by high mobility, intermittent connectivity, and unreliability of the wireless channel. In this paper, a novel adaptive distributed cooperative medium access control (ADCMAC) protocol is proposed in order to address the inherent problems in the IEEE 802.11 standard when employed in vehicular networks. ADC-MAC exploits spatial diversities to maximize the system throughput as well as the service range of vehicular networks. This is accomplished through adaptively selecting the most suitable helper and transmission mode for transmit/receive pairs among direct transmission (DT), cooperative relay (CR) transmission and two-hop relay (TR) transmission, in accordance with the channel quality and the positioning of relay nodes. Both our Markov Chain modeling based theoretical analysis and ns-2 simulation experiments show that our ADC-MAC protocol outperforms existing schemes under the same network scenarios and maximizes the achieved system throughput and service distance.
\end{abstract}

Index Terms-MAC Protocol, Cooperative Relaying, Vehicular Networks.

\section{INTRODUCTION}

$\mathbf{V}$ EHICULAR Networks are a cornerstone of the envisioned Intelligent Transportation Systems (ITS) by enabling vehicles to communicate with each other via InterVehicle Communication (IVC) as well as with roadside base stations via Roadside-to-Vehicle Communication (RVC) [1]. In the foreseeable future, most new vehicles will be equipped with wireless radio devices capable of IVC and RVC to contribute to a safer and more efficient driving experience. These systems will provide time-sensitive and location-aware information to drivers and other vehicles from localized traffic updates to warning signals when the car ahead abruptly brakes. Such timely information conveyed over a vehicular network is envisioned to help significantly reduce the number of annual traffic fatalities and injuries [2].

Today, wireless LANs based on 802.11 [3] have become a de facto standard and are in use as a wide-area wireless technology due to unlicensed radio spectrum, low-cost

Manuscript received 5 January 2010; revised 7 May 2010 and 12 July 2010. This work was supported by a grant from the Federal Railroad Administration (FRA) under Grant 25-1105. The support provided to us by BNSF and Union Pacific Railway.

The authors are with the Telecommunications Engineering Laboratory (TEL), Department of Computer and Electronics Engineering, University of Nebraska-Lincoln, Omaha, NE 68182 USA (e-mail: \{tzhou, hsharif, mhempel, pmahasukhon, wwang, tma\}@unlnotes.unl.edu).

Digital Object Identifier 10.1109/JSAC.2011.110108.

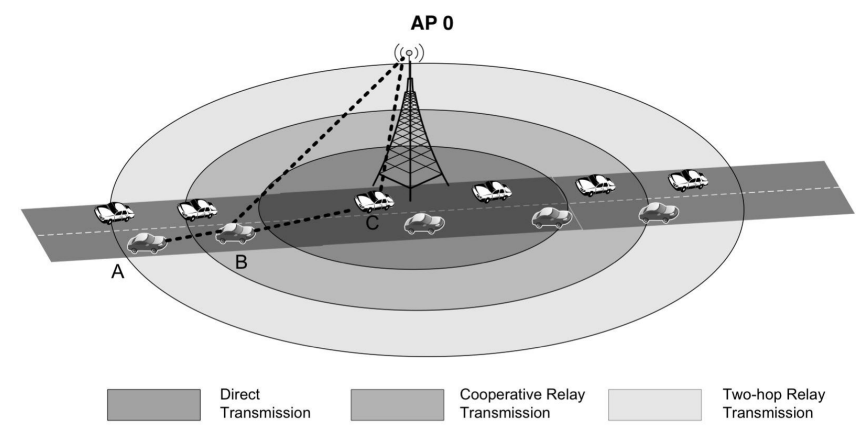

Fig. 1. Application scenario

equipment and end-to-end IP connectivity. However, these networks typically suffer from having smaller cell coverage areas than EDGE and WCDMA systems [4]. With the same transmit power and antenna gain, its range is just one-third that of the later two competing cellular network technologies. Additionally, other critical challenges exist when wireless LANs are used to establish vehicular networks [5]:

1) Time sensitivity: time-sensitive data must be collected, processed and disseminated within a given time window. If this cannot be guaranteed by the deployed network technology, data will become stale and has to be treated as invalid due to its critical nature;

2) Location awareness: both data gathered from vehicles and data consumed by vehicles are highly locationdependent. Systems that operate in a vehicular network environment thus need to be able to correlate information to location;

All of the above requirements for vehicular networks indicate the necessity for wireless LANs to support robust realtime high-speed data communications. A concrete example is shown in Fig. 1: vehicles $\mathrm{C}$ can communicate with its service access point (AP 0) directly to get the maximum throughput, while vehicle $\mathrm{B}$ can only achieve its maximum throughput with the assistance of vehicle $\mathrm{C}$. Even worse, without the relay service provided by vehicle $\mathrm{B}$, vehicle A cannot communicate at all with AP 0. However, the standard IEEE 802.11 protocol cannot provide cooperative relaying or dynamic inter-vehicle relaying services to meet this requirement of vehicular networks.

Therefore, the motivation of our research is to design a new distributed cooperative relay MAC protocol to maximize the vehicles' achieved system throughput and service distance by adaptively selecting among suitable transmission modes and relay nodes according to the channel quality between the transmit/receive node pair and the layout of relay nodes within 


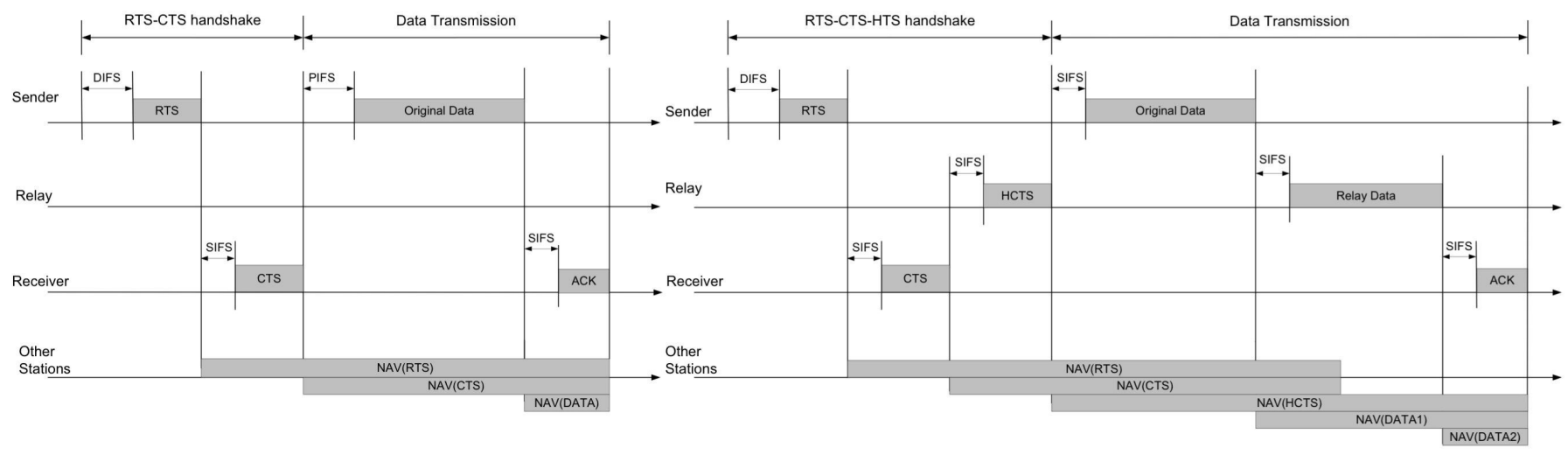

(a) Direct DATA Transmission

(b) Cooperative Relay DATA Transmission

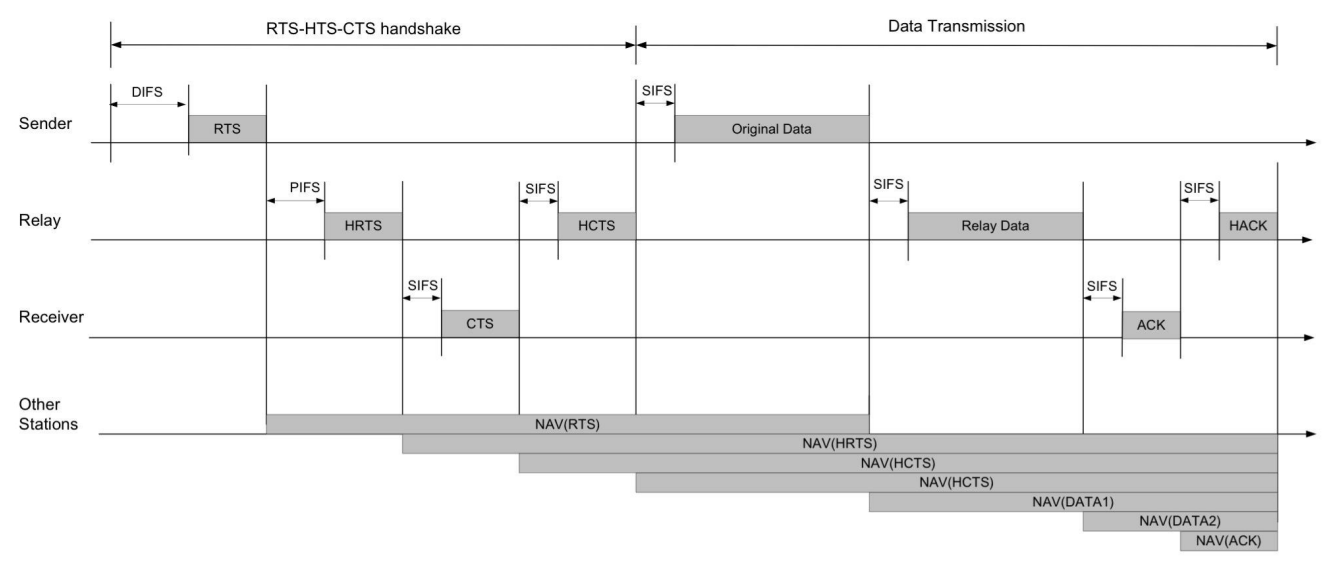

(c) 2-hop Relay DATA Transmission

Fig. 2. Timeline of ADC-MAC protocol

the communication area. Most importantly, our proposed MAC protocol is backward-compatible with the standard IEEE 802.11 protocol, so that it can be deployed in coexistence scenarios side-by-side with traditional IEEE 802.11 networks and cooperative-relay enhanced vehicular wireless networks.

In this paper, a novel fully adaptive distributed cooperative medium access control (ADC-MAC) protocol is proposed for vehicular networks. Our approach leverages new handshaking messages: Helper-Request-To-Send (HRTS) and Helper-ClearTo-Send (HCTS), which are used for cooperative relay activity coordination. With this RTS-CTS-HRTS/HCTS triangular handshake, the transmit/receive pair can now choose the most suitable helper for assistance during data transmissions.

The key features of our proposed ADC-MAC include:

1) This protocol is fully distributed and does not depend on time synchronization among nodes within the entire network. This overcomes a significant shortcoming of traditional relay approaches, which depend on additional devices, such as a global positioning system (GPS) and thus increase the cost of the deployed infrastructure;

2) ADC-MAC can self-learn the layout information of relay nodes by heartbeat broadcast among mobile stations. Predetermined knowledge is not necessary in our proposed approach, greatly simplifying the network operation complexity;
3) The relaying path and mode is decided by ADC-MAC, which is typically decided at the routing layer in the traditional workings of the network stack. By this way, ADC-MAC can be adaptive to high-mobility scenarios undergoing fast fading.

The remainder of this paper is structured as follows. In Section II, a description of related research studies is provided. An overview of our proposed protocol is presented in Section III. Our protocol and its design details to support mobility are described in Section IV. The theoretical analysis and simulation validation are presented in Section $\mathrm{V}$ and VI. Finally, section VII concludes the paper.

\section{RELATED WORK}

Wireless LANs can provide multiple data rates for transmissions. For example, $802.11 \mathrm{~b}$ supports $1 \mathrm{Mbps}, 2 \mathrm{Mbps}, 5.5 \mathrm{Mbps}$ and $11 \mathrm{Mbps}$ data rates with the lowest data rate of $1 \mathrm{Mbps}$ being designated as the "basic data rate" that is used for achieving maximum service range. Under the same channel quality and transmit power, a lower data rate exhibits a lower bit error rate (BER) than higher data rates. Wireless stations that suffer from poor channel quality tend to use lower data rates to reduce the BER of each transmission and thus increase the success rate of packet delivery. However, reducing the data 
rate typically also results in performance degradation for the entire network [6].

The basic idea of cooperative relaying is that a wireless station with low data rate can be assisted by neighboring stations with higher rates for its transmissions. These assisting nodes are referred to as relay nodes or helper stations. With such assistance available, the low rate station will be able to transmit data with a higher rate to the relay node which in turn will forward the data to the destination with a higher rate. This achieves an overall higher performance than if the low rate station were to transmit the data directly to the destination.

Only few research studies focus on the cooperative MAC protocol design. Relay-enabled DCF (rDCF), which is a DCFbased MAC protocol, is proposed in [7] to exploit the multirate capability of IEEE 802.11. Based on the channel condition among mobile nodes, rDCF can intelligently apply two-hop data transmission to achieve higher transmission rate utilizing a triangular handshake among a source node, a destination node and a predetermined relay node. The location requirement for useful relay nodes is also analyzed in this paper. CoopMAC I and II are introduced in [8,9] and are also implemented on a Linux testbed. The author also studied the impact of cooperation on inter-cell interference. In CoopMAC, each mobile station and access point (AP) maintains a table, referred to as CoopTable, which includes candidate helpers that can be used for assistance during transmission. However, each CoopTable needs to be configured manually.

Cooperative MAC protocol (COMAC) is proposed in [10]. It enables cooperation in a realistic scenario using $802.11 \mathrm{~g}$ radios with a single predetermined relay node, [11] extends the work of [10] by using predetermined K ACO (Availableto-Cooperate) steps to select the best relay from a set of candidate relay nodes.

All of the aforementioned studies conducting cooperative communication depend on predetermined knowledge to configure and select relay nodes for transmit/receive pairs. Hence these schemes cannot sufficiently exploit spatial diversity.

For selecting a suitable relay node dynamically, another relay-enabled MAC protocol is proposed in [12]. After receiving RTS and CTS packets, the helper transmits ready-torelay (RTR) packets to declare that it has the ability to relay. Alternatively, it will refrain from sending RTR if it determined that it is not suitable for relaying the pending message. In this scheme, however, if there is more than one suitable relay node a collision will unavoidably occur, which is detrimental to the overall network performance.

Vehicular cooperative media access control (VC-MAC) protocol, proposed in [13], is designed for AP-Downlink scenarios, which assume that all vehicles within the AP's range can be synchronized by receiving the data packet from that AP. By utilizing two specific intervals for information exchange and relay set selection, VC-MAC can then select the most suitable relay node to relay packets. However because for transmitting a packet the whole network needs to synchronize once, the overhead of VC-MAC is too high and in the worst case only about 10 percent of the total bandwidth can be used to transmit data.

Therefore the relay node selection schemes shown in the above both approaches are not optimal for vehicular networks,

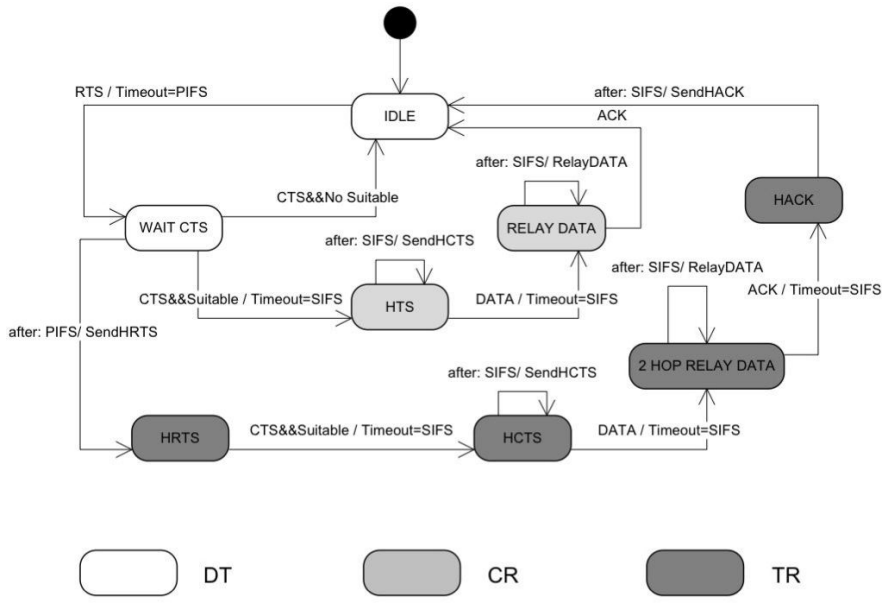

Fig. 3. The state machine of ADC-MAC protocol

especially for heavy traffic loads and high spatial diversity.

Other relative work includes [1] and [14]. The main observation in [1] indicates that the performance of cooperative relaying is appreciably degraded by transmission directionality, such as directional antennas with a sufficiently narrow beam, while [14] theoretically analyzed the performance of the cooperative relaying MAC protocol by Markov chain.

\section{Proposed PRotocol}

This section presented the design details on the proposed cooperative relaying protocol. ADC-MAC is designed to be backward-compatible with IEEE 802.11 MAC. In the original IEEE 802.11 standard, if a packet goes to a node that is not its intended destination, the node will ignore the packet and sets its Network Allocation Vector (NAV) to the value of the duration field specified in the packet header. The NAV specifies the earliest point at which the station can try to access the medium again. The values of the duration field of each packet type are shown in TABLE-I,

The communication timeline for DT, CR and TR modes in the proposed ADC-MAC protocol are shown in Fig. 2, while the internal finite state machine(FSM) of the proposed ADCMAC protocol are shown in Fig. 3, which is expressed by Unified Modeling Language(UML). There are 8 internal states and 15 external events defined in this FSM for normal packet processing. For abnormal case processing, the timer will be set in accordance with the duration values of the packet header. When timeout, the FSM shall be reset to the "IDLE" state.

Our proposed MAC protocol includes two stages:

1) Three party handshake stage and

2) Data transmission stage.

The first stage is used to select the most suitable transmission mode and the most suitable helper. The packet format modifications in ADC-MAC for each packet type are shown in fig. 4.

Three party handshake: All packets in this period are transmitted with the basic data rate. The transmitter carries out the physical carrier sensing to detect the idle state of the channel. If the received power is constantly lower than the given threshold for a contention interval, it will consider the 


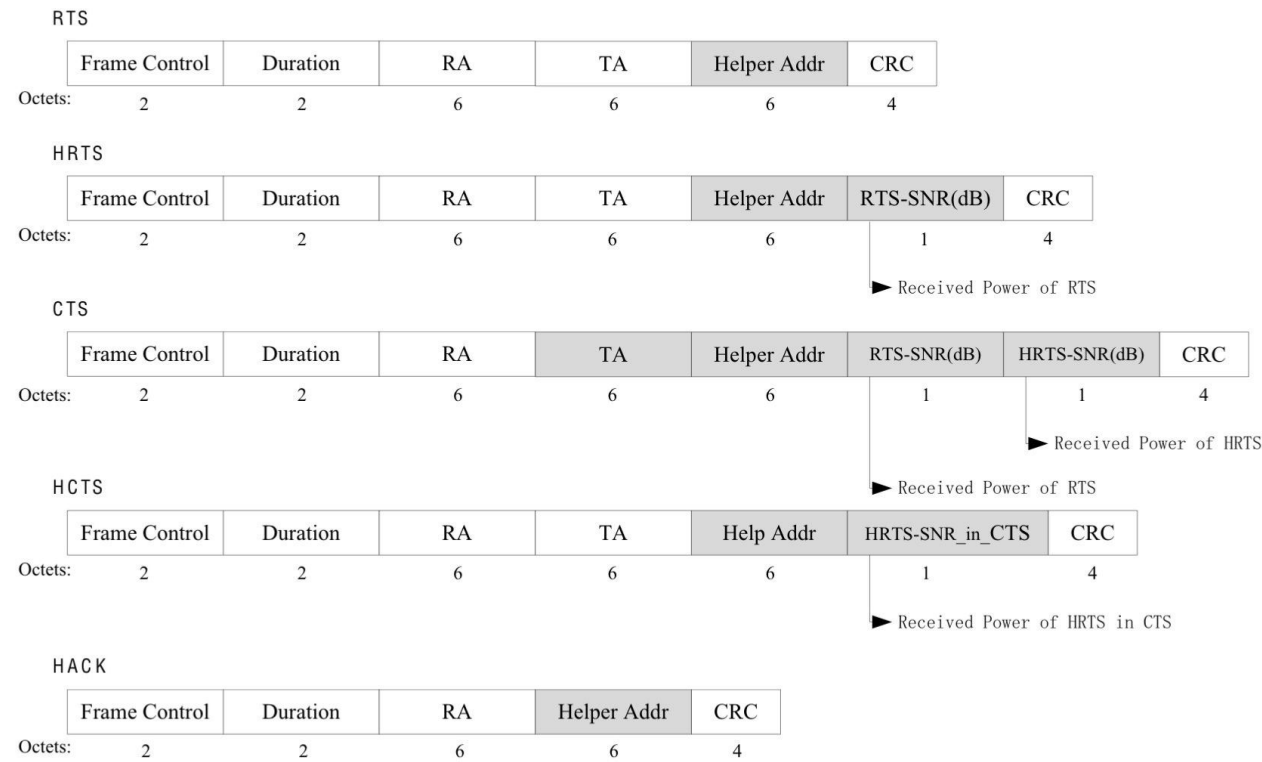

Fig. 4. Modified control packet format of ADC-MAC

channel to be idle and send an RTS packet to the receiver. The RTS packet contains a duration field and reports the transmitting time for the following DATA frame. According to the value in this field, other stations setup the corresponding entry in their NAV table. Immediately upon receiving this RTS packet, the receiver will respond with a CTS packet back to the sender. One additional Signal-to-Noise ratio (SNR) field in the CTS packet format is introduced to report the received SNR (dB) value of the RTS packets.

Through this RTS-CTS handshake, the transmitter can exploit diversity gain and choose a suitable transmit power to send DATA frames. The above procedure is similar to that presented in the IEEE 802.11 protocol standard. However, when a transmitter receives the corresponding CTS packet with the proposed protocol it will transmit a DATA packet starting immediately after expiration of a PIFS time interval, instead of after an SIFS interval in the IEEE 802.11 standard. After the PIFS interval expires, and if the channel is still idle, the transmitter will start to transmit data. Therefore, if there is no suitable relay node available or if none is needed, the ADC-MAC protocol only introduces an additional time interval overhead at an interval of |PIFS-SIFS|. If an idle relay node can receive both RTS and CTS packets, its MAC address matches the helper address field in the CTS packet, and both received SNR values are greater than the given threshold, it will declare that it has the ability to relay data by transmitting an HCTS packet. When the transmitter receives this HCTS packet, it will evaluate both the RTS-SNR field and the CTSSNR field of this message. The RTS-SNR field contains the SNR (dB) of the RTS packet received at the receiver node and reported by it in the corresponding field in the CTS message. The CTS-SNR field contains the measured SNR (dB) of the CTS message observed by the relay node. The relay node therefore measures the SNR of the CTS message directly, extracts the RTS-SNR value from the CTS message, and then reports both values in the HCTS message back to the transmitter. If both the value of RTS-SNR field and the

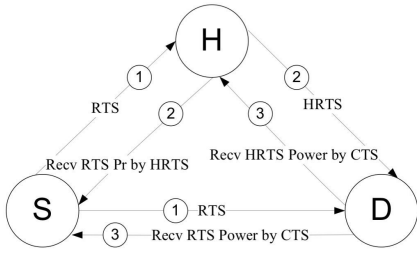

(a)

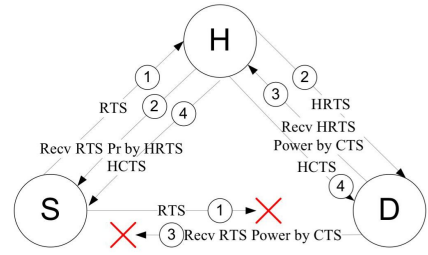

(b)
Fig. 5. Three party handshakes among the source, the destination and the helper node

value of the CTS-SNR field are greater than the given RelaySNR threshold, the transmitter will choose this relay node as the helper; otherwise it will transmit the DATA packet to the receiver directly.

Fig. 5-a shows the case that the receiver can get the RTS packet from the transmitter directly, while Fig. 5-b shows the case that if the RTS packet is missing at the receiver side, the relay node need to help the receiver forward HRTS-SNR information to the transmitter by broadcasting a HCTS packet. This mechanism in effect provides an extended coverage area through relaying between transmitter and receiver.

By the above three-party handshake process, ADC-MAC overcomes the hidden node problem in 2-hop partly connected networks, because all nodes can hear the HRTS/HCTS packets sent by the middle relay nodes to set up the right NAV values.

In ADC-MAC, for each data transmission, the suitable helper is nominated by the transmitter, and validated by the above three-party handshake process. The helper candidate selection algorithm is described in the next section.

Data transmission: After completion of the triangle handshake procedure, the transmitter can initiate data transmission immediately. The transmitter chooses the most suitable transmission model among direct transmission, cooperative relay transmission and two-hop relay transmission. It also designates the most suitable helper to relay data, if needed. In the twohop mode, if the relay node receives ACK from the receiver, 
it will transmit a HACK to the transmitter. From the HACK sent by the helper, the transmitter will know that the current data transmission is successful. All nodes have to wait for DIFS plus their respective backoff time to start transmitting new data.

Overall, the above ADC-MAC protocol design utilizes the characteristics of vehicular networks and takes into account the impact of spatial diversity by introducing the RTS-CTSHRTS/HCTS handshake procedure outlined above.

\section{OPtimization FOR Network MOBility}

We can characterize vehicular networks as environments in which all nodes except roadside base stations are mobile and exhibit unpredictable mobility patterns only loosely constrained by the routes and lanes provided by the road infrastructure.

In ADC-MAC, each node maintains a table, referred to as CoopTable, to save the latest received RSSI values of other nodes, which are directly measured or reported by its neighbors using heartbeat packets. The original idea of CoopTable is from CoopMAC [8]. The key innovation of our CoopTable is self-learning, whose contents can be dynamically updated by heartbeat packets and the three-party handshake processes.

By listening to periodic heartbeat packets, a node can detect the mobility pattern of its neighbors. Because all nodes except roadside base stations are mobile, the content of each CoopTable is impossible to be predetermined or configured manually for each node. Therefore, in ADC-MAC, each node is required to collect its neighbor information by receiving heartbeat packets, and control packets in the three-party handshake process to build up its CoopTable.

In the remainder of this section, we describe the details of the issues mentioned above. These functionalities are designed to support the mobility of vehicular network environment.

\section{A. Heartbeat Broadcasting}

Every node periodically broadcasts heartbeat packets. The format of heartbeat packet payload is shown in Fig. 6 and includes its latest collected neighbor information. Each node first calculates the average SNR value of all sub-entries for each individual CoopTable entry. If a sub-entry's highest SNR value is greater than the given threshold and its flag field is "dirty", the MAC address, SNR value and the latest timestamp of this CoopTable entry will be included in a heartbeat packet.

\section{B. CoopTable Self-Learning}

The entry structure of the CoopTable is shown in Fig. 7. Each entry is indexed based on the MAC address of the corresponding node and includes multiple sub-entries. Each sub-entry records the last $\mathrm{K}$ number of received RSSI values and timestamps from a single helper. The index of a subentry is the MAC address of the helper node. If subentry index is the same with the entry index, it indicates that the RSSI values are received from that node directly. When a new received SNR value is inserted into the CoopTable the flag of the corresponding sub-entry will be set to "dirty". As soon
TABLE I

The Value of Duration Field in Each Packet Type

\begin{tabular}{|c|c|}
\hline Packet Type & Duration Value \\
\hline$\overline{R T S}$ & $\begin{array}{l}\text { SIFS+T[CTS }]+P I F S+T[D A T A]+ \\
\text { SIFS+T[ACK }]+3 \sigma\end{array}$ \\
\hline$C T S$ & $\mathrm{PIFS}+\mathrm{T}[\mathrm{DATA}]+\mathrm{SIFS}+\mathrm{T}[\mathrm{ACK}]+2 \sigma$ \\
\hline$\overline{D A T A}(D T)$ & $\mathrm{SIFS}+\mathrm{T}[\mathrm{ACK}]+\sigma$ \\
\hline $\operatorname{HCTS}(\mathrm{CR})$ & $\begin{array}{l}\text { SIFS+T[DATA }]+ \text { SIFS+T[DATA }]+ \\
\text { SIFS+T }[\mathrm{ACK}]+3 \sigma\end{array}$ \\
\hline$\overline{D A T A}$ (CR-Original) & SIFS+ T[DATA $]+$ SIFS+T[ACK $]+2 \sigma$ \\
\hline DATA(CR-Relay) & $\mathrm{SIFS}+\mathrm{T}[\mathrm{ACK}]+\sigma$ \\
\hline HRTS & 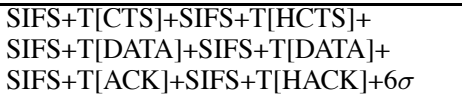 \\
\hline$\overline{H C T S}(T R)$ & $\begin{array}{l}\text { SIFS+T[DATA }]+ \text { SIFS+T[DATA }]+ \text { SIFS+ } \\
\text { T[ACK }]+ \text { SIFS }+\mathrm{T}[\mathrm{HACK}]+4 \sigma\end{array}$ \\
\hline DATA(TR-Original) & $\begin{array}{l}\text { SIFS+T[DATA }]+ \text { SIFS+T[ACK }]+ \text { SIFS+ } \\
\text { T[HACK }]+3 \sigma\end{array}$ \\
\hline$\overline{D A T A}(\mathrm{TR}$-Relay $)$ & SIFS+T[ACK $]+$ SIFS+T[HACK $]+2 \sigma$ \\
\hline $\operatorname{ACK}(T R)$ & $\mathrm{SIFS}+\mathrm{T}[\mathrm{HACK}]+\sigma$ \\
\hline
\end{tabular}

Note: $\sigma$ is the maximum one-way propagation delay, where T [.] is the expected packet transmit time. DT: direct transmission; CR: cooperative relaying; TR: two-hop relaying.

as a sub-entry is used for heartbeat broadcasts, its flag field will be restored to "clear". To reduce network overhead, only sub-entries with the flag value "dirty" will be involved in the heartbeat broadcasts.

\section{Helper Candidate Selection Algorithm}

By heartbeat broadcasting, a mobile node can know the information of the whole network from its neighbors then build up its CoopTable dynamically. For each data transmission, it need to choose a suitable transmission mode among DT, CR and TR and a suitable node to relay data if needed.

The best helper candidate selection problem can be solved by the shortest path algorithm. This is more efficient. For example, Dijkstras algorithm can find the shortest paths in $O|V|^{2}$, where $\mathrm{V}$ is the number of vertices in the graph. We convert this problem into finding the shortest paths as follows: We construct a directed graph $D(V \cup T \cup R, E)$, which $\mathrm{V}$ is the set of helper candidate, $\mathrm{T}$ and $\mathrm{R}$ are the transmitter and the receiver node. $\mathrm{E}$ is the set of edges among them. The reciprocals of the data rate between two nodes are used as the length of edges in graph D. By this way, we can find the shortest path between $\mathrm{T}$ and $\mathrm{R}$ in graph $\mathrm{D}$. The vertex along this path is chosen as the suitable helper candidate. Fig. 8 shows the convention process.

\section{TheOretical AnAlysis}

In this section, the theoretical throughput and delay performance of the proposed ADC-MAC protocol is evaluated based on Bianchi's Markov chain based model, which mathematically describes the performance of the IEEE 802.11 Distributed Coordination Function (DCF) protocol [15-18]. The influences of backoff, packet length, total station number, retransmission limitation, collision probability, and channel bit error rate are taken into consideration in our modeling.

To facilitate our discussion and keep the tractability of the analytical model of the IEEE 802.11 protocol performance, we make the following assumptions: 

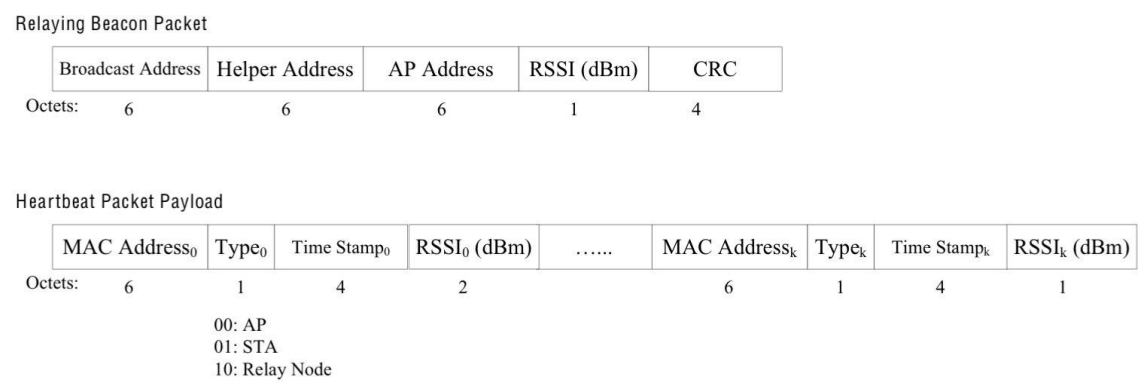

Fig. 6. Packet formats of the heartbeat packets

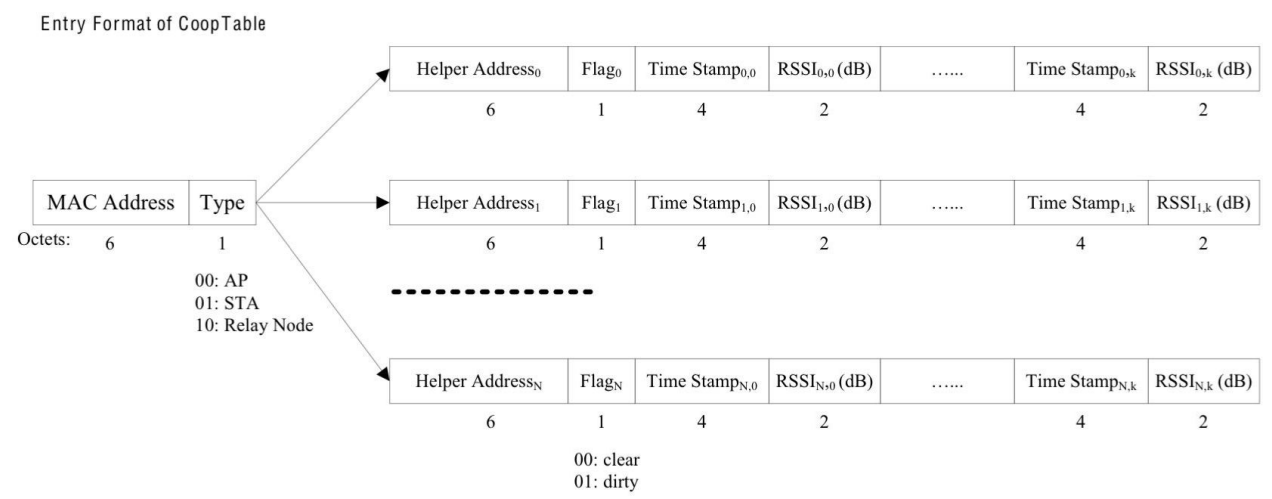

Fig. 7. Entry structure in CoopTable

1) Each node only has one MAC interface, which cannot transmit and receive at the same time;

2) The packet transmissions of all nodes share the same channel;

3) The network will consist of a single source, a destination and some potential helper nodes. This role assignment is time varying;

4) The MAC header and the data payload are transmitted with data rate $R_{d}$ (in bits per second) while the physical layer preamble, management and control packets (i.e., Beacon, RTS, CTS, HRTS, HCTS, HACK and ACK packets) are transmitted with basic data rate $R_{c}$ (in bits per second);

5) The channel is prone to error and the channel noise is assumed to be white. Therefore the channel bit error $P_{b}$ is uniformly distributed with error events and independent of each other;

6) There is no error correction mechanism in the physical layer;

7) The SNR decision thresholds for selecting between relaying transmissions and direct transmission are known by all nodes;

8) When receiving a packet, the receiver can also obtain physical layer information of that packet, such as received power, noise floor, channel index, by reading corresponding registers similar to the functionality provided by Atheros MAC chipsets [19];

9) Each node maintains a CoopTable, which saves neighbor information and helps the node to make relaying decisions;

10) Each node transmits packets with probability $\tau$;

11) Omni-directional antennas are employed.

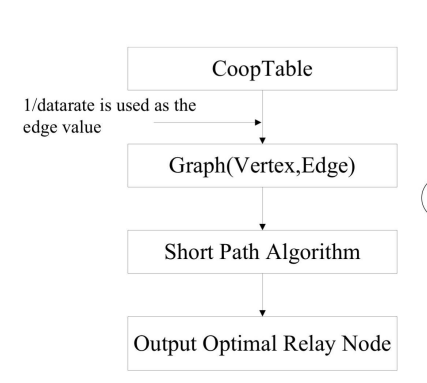

(a)

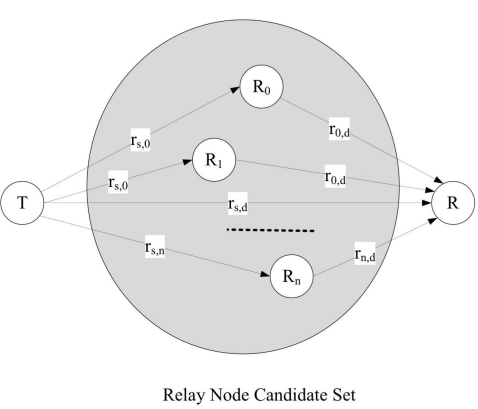

(b)
Fig. 8. Dynamic helper candidate selection

In the rest of this section, firstly the static performance of ADC-MAC is evaluated by our Markov chain based modeling, and then its control overhead is quantitatively analyzed.

\section{A. Performance Analysis}

The normalized throughput $\mathrm{S}$ can be expressed as the ratio

$$
S=\frac{E[p]}{E[\operatorname{slot}]}
$$

where $E[$.$] is the expectation operator, E[p]$ is the average transmitted payload length in a slot time and $E[$ slot $]$ is the average length of a slot time.

For our proposed protocol, $S$ can therefore be expressed as:

$$
S=\frac{P_{1} P_{s}\left(1-P_{3}\right) T_{p}}{\left(1-P_{1}\right) \sigma+P_{1} P_{s} T_{s}+P_{1}\left(1-P_{s}\right) T_{c}}
$$

where $T_{P}$ is the average payload transmit time, $T_{s}$ and $T_{c}$ are the average time of a successful transmission and a collision, respectively, and $\sigma$ is the duration of a slot time. 

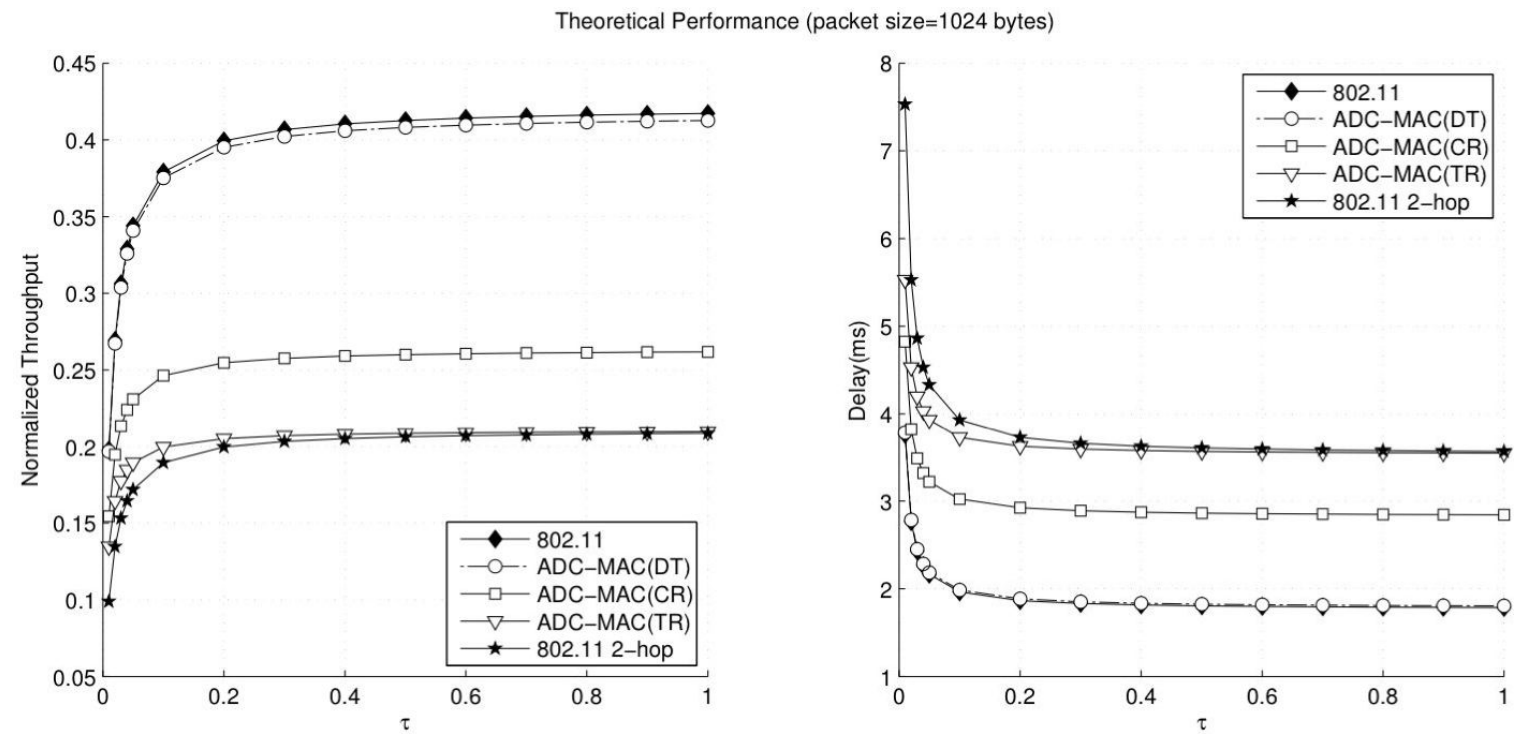

Fig. 9. Theoretical performance comparison between ADC-MAC and IEEE 802.11 protocol under different probability
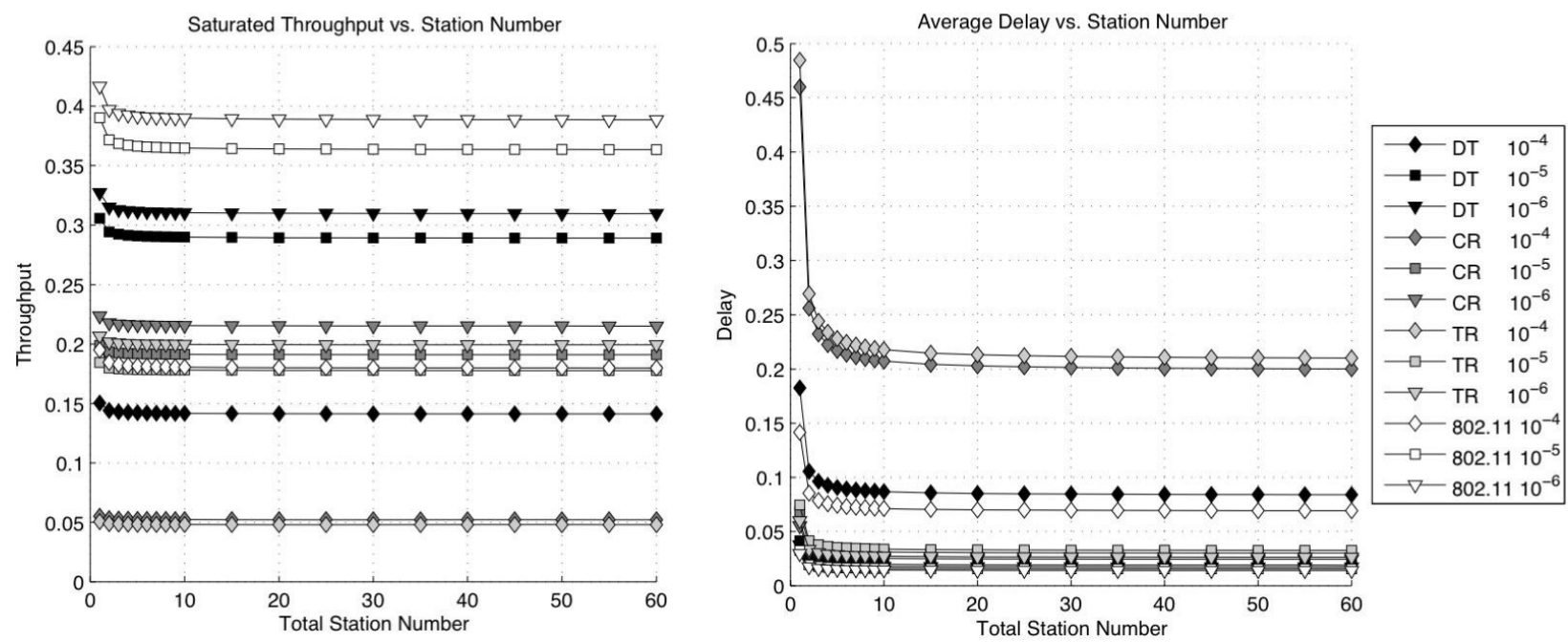

Fig. 10. The saturated throughput comparison between each mode in ADC-MAC protocol and 802.11 protocol under different BERs with different station numbers

Let $P_{1}$ be the probability that the channel is busy. This means that there is at least one transmission in the considered slot time. If each of the $n$ stations transmits a packet with probability $\tau$, we can get the following equation:

$$
P_{1}=1-(1-\tau)^{n}
$$

Let Ps be the probability of a non-collision transmission.

$$
P_{s}=\frac{n \tau(1-\tau)^{n-1}}{1-(1-\tau)^{n}}
$$

Let $P_{3}$ be the probability that the transmission failure is caused by channel bit errors. Since we assume that channel bit errors are uniformly distributed and error events are independent we can express $P_{3}$ for our ADC-MAC protocol for the cases of direct transmission, cooperative relaying and 2-hop relaying, respectively, as:

$$
P_{3-D T}=1-\left(1-P_{b}\right)^{L_{R T S}+L_{C T S}+L_{D A T A}+L_{A C K}}
$$

$$
\begin{aligned}
& P_{3-C R}=1- \\
&\left(1-P_{b}\right)^{L_{R T S}+L_{C T S}+L_{H C T S}+L_{D A T A}+L_{D A T A}+L_{A C K}}
\end{aligned}
$$

$P_{3-T R}=1-$

$\left(1-P_{b}\right)^{L_{R T S}+L_{H R T S}+L_{C T S}+L_{H C T S}+2 L_{D A T A}+L_{A C K}+L_{H A C K}}$

Furthermore, $T p, T s$ and $T_{c}$ are given as

$$
\begin{aligned}
& T_{p-D T}=\frac{L_{D A T A}}{R_{c}} \\
& T_{p-C R}=T_{p-T R}=\frac{2 L_{D A T A}}{R_{d}} \\
& T_{s-D T}=D I F S+2 S I F S+P I F S+ \\
& \frac{L_{R T S}+L_{C T S}+L_{A C K}}{R_{c}}+\frac{L_{D A T A}}{R_{d}} \\
& T_{s-C R}=D I F S+4 S I F S+ \\
& \frac{L_{R T S}+L_{C T S}+L_{H C T S}+L_{A C K}}{R_{c}}+\frac{2 L_{D A T A}}{R_{d}} \\
& T_{s-T R}=D I F S+P I F S+6 S I F S+\frac{2 L_{D A T A}}{R_{d}}+ \\
& \frac{L_{R T S}+L_{H R T S}+L_{C T S}+L_{H C T S}+L_{A C K}+L_{H A C K}}{R_{C}}
\end{aligned}
$$




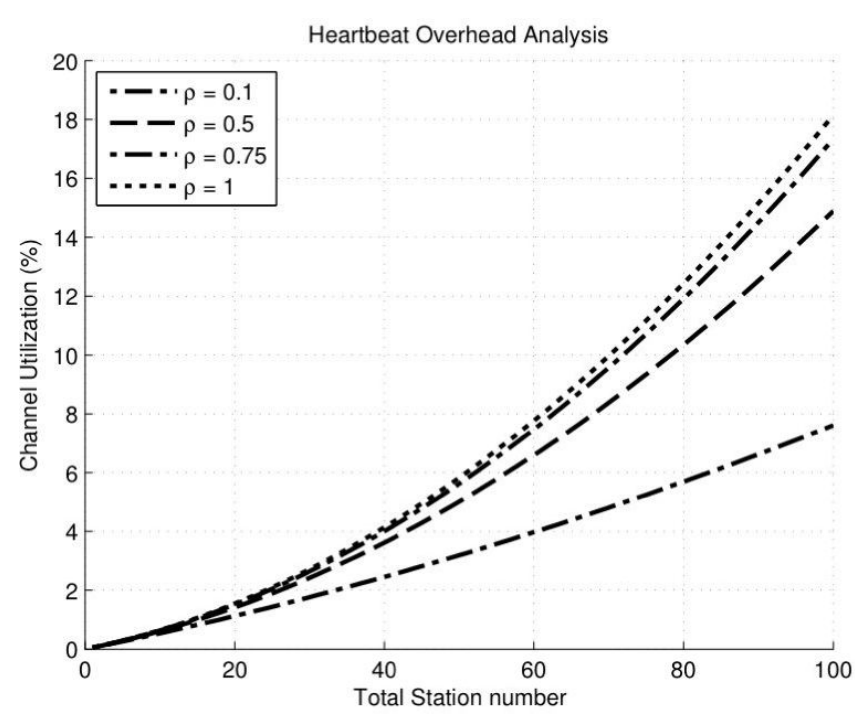

Fig. 11. The heartbeat overhead vs. total station number for different values

$$
T_{c}=D I F S+S I F S+\frac{L_{R T S}}{R_{c}}
$$

where $L_{R T S}, L_{C T S}, L_{D A T A}, L_{A C K}, L_{H R T S}, L_{H C T S}$ and $L_{H A C K}$ are the length of the RTS, CTS, DATA, ACK, HRTS, HCTS, HACK packets, respectively.

$E\left[D_{\text {int }}\right]$, the average packet interval time between two successfully received packets at one receiver, can be obtained from the throughput expression by substituting equation (2) :

$$
E\left[D_{\text {int }}\right]=\frac{n \cdot E[p]}{S}=n \cdot E[\text { slot }] .
$$

By combining this expression with equations (3) and (4), the above equation can be rewritten as:

$$
E\left[D_{\text {int }}\right]=\frac{E[\text { slot }]}{\tau(1-\tau)^{n-1}\left(1-P_{3}\right)} .
$$

The average time to drop a packet can be expressed as:

$$
E\left[D_{\text {drop }}\right]=E\left[X_{d r o p}\right] \cdot E[\text { slot }]
$$

where $E\left[X_{d r o p}\right]$ is the average number of slot times for a dropped packet after $m$ retransmissions.

Due to the fact that $\mathrm{p}_{\mathrm{e}}^{\mathrm{m}+1}$ is the probability that a packet will finally be dropped after $m$ retransmissions, and the average number of time slots spent in the backoff stage $j$ is equal to $\frac{\mathrm{W}_{\mathrm{j}+1}}{2}, E\left[X_{d r o p}\right]$ can be expressed as:

$$
E\left[X_{d r o p}\right]=\sum_{j=0}^{m} \frac{W_{j}+1}{2}
$$

When $\frac{\mathrm{p}_{\mathrm{e}}^{\mathrm{m}+1}}{1-\mathrm{p}_{\mathrm{e}}^{\mathrm{m}+1}}$ is the average number of dropped packets relative to a successful transmission, the average packet delay can be expressed as:

$$
E[D]=E\left[D_{i n t}\right]-\frac{P_{e}^{m+1}}{1-p_{e}^{m+1}} E\left[D_{d r o p}\right]
$$

The modeling of the performance of IEEE 802.11 protocol can be found in [20]. Two-hop 802.11 protocol is treated as

\begin{tabular}{|c|c|}
\hline Parameter & Value \\
\hline Data Rate (Mbps) & $\begin{array}{l}1,2,5.5,11 \text { (basic } \\
\text { rate }=1 \mathrm{Mbps} \text { ) }\end{array}$ \\
\hline SlotTime $(\mu s)$ & 20 \\
\hline DIFS, PIFS, SIFS $(\mu s)$ & $50,30,10$ \\
\hline ACK, RTS, CTS, HRTS, HCTS, HACK (bytes) & $14,26,20,27,28,20$ \\
\hline PLCP length (bits) & 96 \\
\hline $\mathbf{C} W_{\min }$ & 31 \\
\hline $\mathbf{C} W_{\max }$ & 1023 \\
\hline Heartbeat Interval (second) & 0.1 \\
\hline Receiver sensitivity for $1,2,5.5,11 \mathrm{Mbps}(\mathrm{dBm})$ & $-94,-91,-87,-82$ \\
\hline Transmit Power (W) & 0.03 \\
\hline Antenna Gain of AP, STA (dBi) & 7,5 \\
\hline Path Loss Exponent & 2.8 \\
\hline
\end{tabular}
combining two independent DCF transmissions together.

Assuming $R_{d}$ and $R_{c}$ are $11 \mathrm{Mbps}$ and $1 \mathrm{Mbps}$, respectively, we can calculate the numerical results of the saturation
TABLE II

SIMULATION PARAMETERS

throughput and delay of ADC-MAC protocol. Fig. 9 shows both the theoretical throughput and delay performance comparison for the IEEE 802.11, and ADC-MAC protocols under different values when the packet size is 1024 bytes. Fig. 10 shows show the theoretical maximum throughput and delay performance in the saturated channel comparison among IEEE 802.11 and each mode in ADC-MAC, when the packet size is 1024 bytes under different BERs. From both figures, it is observed that the performance of DT and TR mode in ADCMAC are very close to those of direct and two-hop 802.11 protocol, respectively. However when the channel is idle, the throughput performance in TR mode of ADC-MAC is higher than that of the two-hop IEEE 802.11 protocol.

\section{B. Control Overhead Analysis}

The control overheads in the proposed ADC-MAC protocol are mainly from heartbeat packets. Because only the subentry with the highest SNR value and the "dirty" flag of a CoopTable entry shall be included in the heartbeat packet, the overhead of heartbeat packets is decided by the channel condition, the mobility pattern, the total station number and the heartbeat interval. In the ideal case: all stations are static and there is no channel fading, because the received SNR values from other nodes is stable, there is no heartbeat packet overhead, while in the worst case: the channel is suffered from fast fading or all nodes are fast moving, the payload length of a heartbeat packet increases linearly with the total station number. So the control overhead of heartbeat packets is:

$$
\begin{aligned}
H_{h}= & \frac{n}{I_{h}}\left[\rho\left(D I F S+\frac{L_{h b}+R_{L} n}{R_{c}}\right)+\right. \\
& \left.(1-\rho)\left(D I F S+\frac{L_{h b}+R_{L} \rho n}{R_{c}}\right)\right] \\
& =\frac{n}{I_{h}}\left[D I F S+\frac{L_{h b}}{R_{c}}+\left(2 \rho-\rho^{2}\right) \frac{R_{L} n}{R_{c}}\right]
\end{aligned}
$$

where $I_{h}$ is the heartbeat interval, $L_{h b}$ is the header length of the heartbeat packet, $R_{L}$ is the length of a station record, and the value of the mobility factor $\rho$ is between 0 and 1 , mean the per cent of the total $\mathrm{n}$ nodes, whose RSSI values are changed in a heartbeat interval. It is obvious that $\rho$ is decided by the channel condition and the mobility patterns. Fig. 11 shows the heartbeat overhead vs. total station number for different $\rho$ values. $\rho=1$ is the worst case, which means that all stations are fast moving or the channel is suffered from fast fading. 


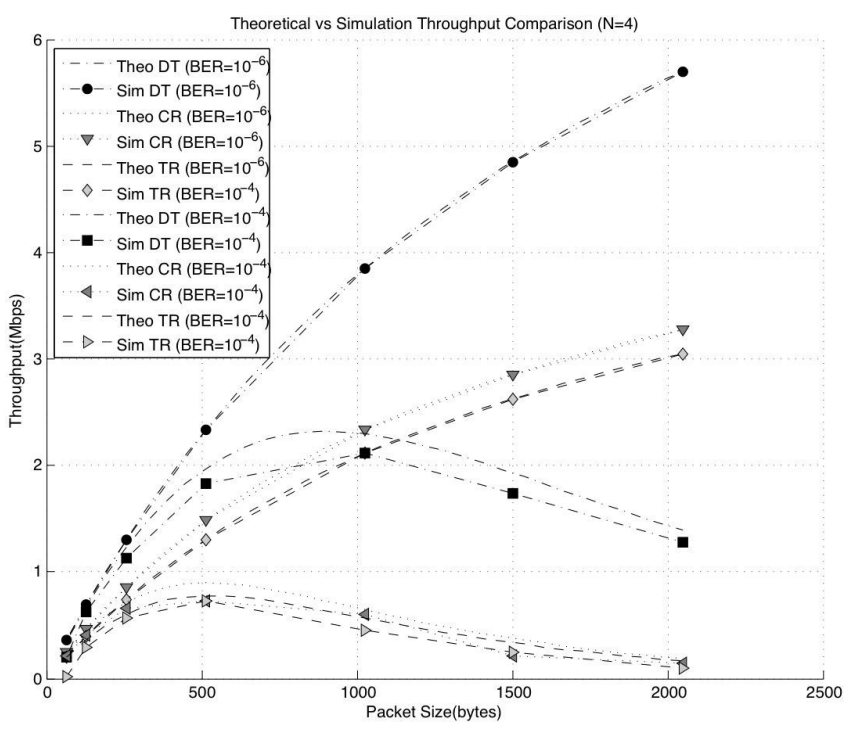

Fig. 12. The throughput comparison between the theoretical analysis and simulation results under different packet sizes.

\section{Simulation Results}

In this section, we show the performance evaluation of the proposed ADC-MAC protocol. The simulation experiments are conducted by ns-2 [20] with version 2.33 , including both stationary and mobile scenarios. The stationary simulation scenario is used to validate the accuracy of our theoretical modeling and evaluate the saturated throughput of each mode in ADC-MAC. The mobile simulation scenario is close to Fig. 1 which includes a roadside fixed AP and multiple mobile stations. It is assumed that there is 1 lane in each direction of the highway and the lane width is 5 meters. The initial locations of vehicles are randomly selected with uniform distribution along the highway. Every vehicle maintains a steady speed, which is also randomly chosen between 40 and $70 \mathrm{mph}$ with uniform distribution.

The main parameters of our simulations are shown in TABLE-II. Both Large-scale propagation module, such as Log-normal shadowing models and small-scale propagation module, such as Ricean fading have been integrated into our simulation to predict the signal power received by the receiver. Log-normal shadowing model simulates the shadow effect of obstructions between the transmitter and receiver, whereas Ricean fading, contributed by [21], is used to describe the rapid changes happening in signal strength over small travel distance or time interval due to multipath propagation.

Fig. 12 shows the throughput performance comparison between the theoretical analysis and simulation results under different BERs. From this graph, it is clear that our Markov chain based theoretical analysis can evaluate accurately the performance of the ADC-MAC protocol. With a higher BER, such as $10^{-4}$, increasing the size of small packets brings better channel utilization. However when the packet size is much larger, the saturation throughput goes down due to a higher frame error rate (FER).

The static saturated throughput comparisons among the three transmission modes of our ADC-MAC protocol and the IEEE 802.11 standard under different packet sizes are shown in Fig. 13. In accordance with our expectation we can find that in the direct transmission mode in ADC-MAC, comparing with IEEE 802.11, only incurs an overhead corresponding to the $|P I F S-S I F S|$ interval. Therefore, the performance of both ADC-MAC and IEEE 802.11 in direct mode is very close. In the cooperative relaying mode, ADC-MAC can achieve higher system throughput than the 2-hop scenario using the IEEE 802.11 protocol, due to the fact that ADCMAC is able to exploit spatial diversity. Finally, in the twohop relaying transmission mode, ADC-MAC can extend the available service range of vehicular networks and still maintain very similar system throughput compared to that of the 2-hop link using the IEEE 802.11 protocol.

The mobility performance comparison between ADC-MAC and 802.11 is shown in Fig. 14. The system throughput and service range of ADC-MAC outperforms the IEEE 802.11 protocol, validating that ADC-MAC can adaptively select the most suitable transmission mode and relay node for data transmission in accordance with to the channel quality between the transmit/receive node pair and the availability and layout of relay nodes within the communication area. Fig. 15 shows the delay performance comparison between TR mode in ADC-MAC and 802.11 protocol with the traditional routing protocol, which is presented by DSDV in the mobile simulation experiments. It is obvious that the end-to end delay in ADC-MAC is much shorter and stable. That is because ADC-MAC decides the relaying path and relay mode for each data transmission by itself without the help of the routing layer, so it can quickly respond to the channel changes due to high-mobility or fast fading. All of the above simulation results validate that our proposed ADC-MAC protocol efficiently utilizes spatial diversity and user diversity, and is therefore able to maximize the system throughput and service range of vehicular networks.

\section{CONCLUSION AND FUtURE WORK}

In this paper, we presented a new adaptive distributed adaptive cooperative MAC protocol: ADC-MAC, which is designed and optimized for vehicular networks to provide robust realtime data communications. Its performance is analyzed by Markov chain based modeling and validated by ns- 2 simulations. Both theoretical analysis and simulation experiments show that ADC-MAC can leverage cooperative communications and exploit spatial and user diversities. Therefore it outperforms the existing protocols under the same channel assumptions and network scenarios. ADC-MAC also provides extended service ranges and a robust wireless communication link in vehicular networks. Integrating ADC-MAC into a real embedded system and developing its security mechanisms are our future research steps.

\section{ACKNOWLEDGMENT}

This study was conducted by the Advanced Telecommunications Engineering Laboratory (TEL) (www.TEL.unl.edu) researchers at the University of Nebraska-Lincoln and it has been funded by the Federal Railroad Administration (FRA) under direction of Terry Tse. This study would not have been 

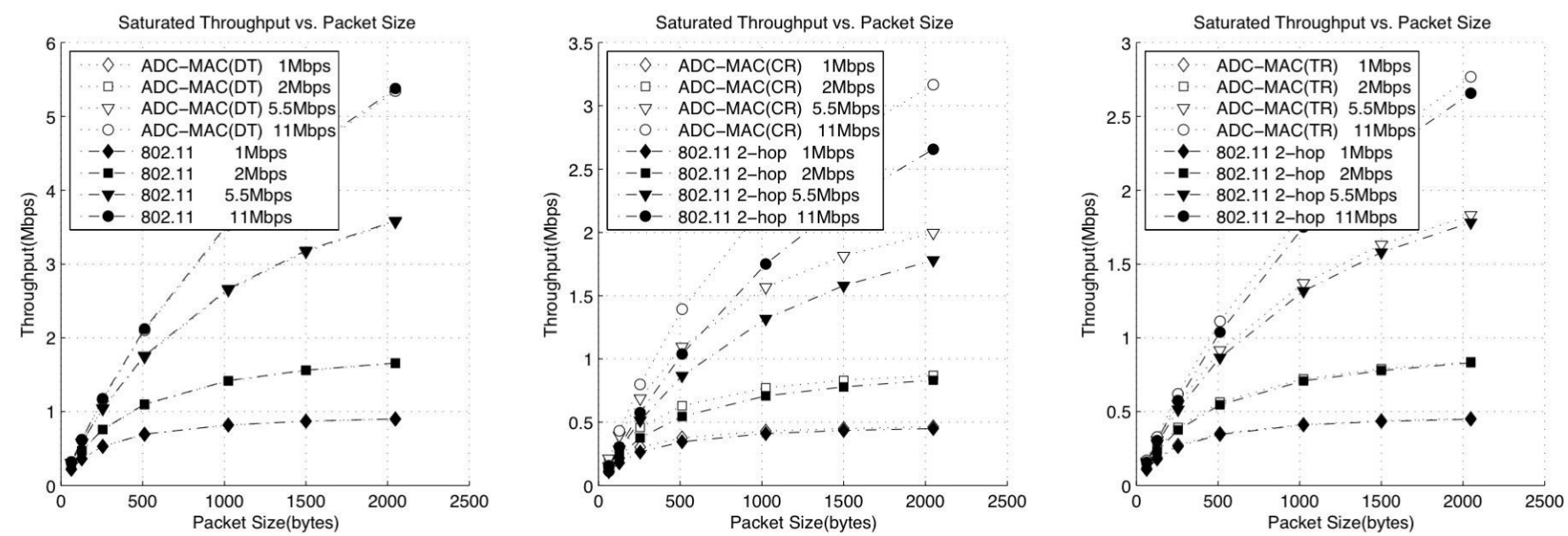

Fig. 13. Saturated throughput comparison between ADC-MAC protocol and 802.11 protocol under different packet sizes

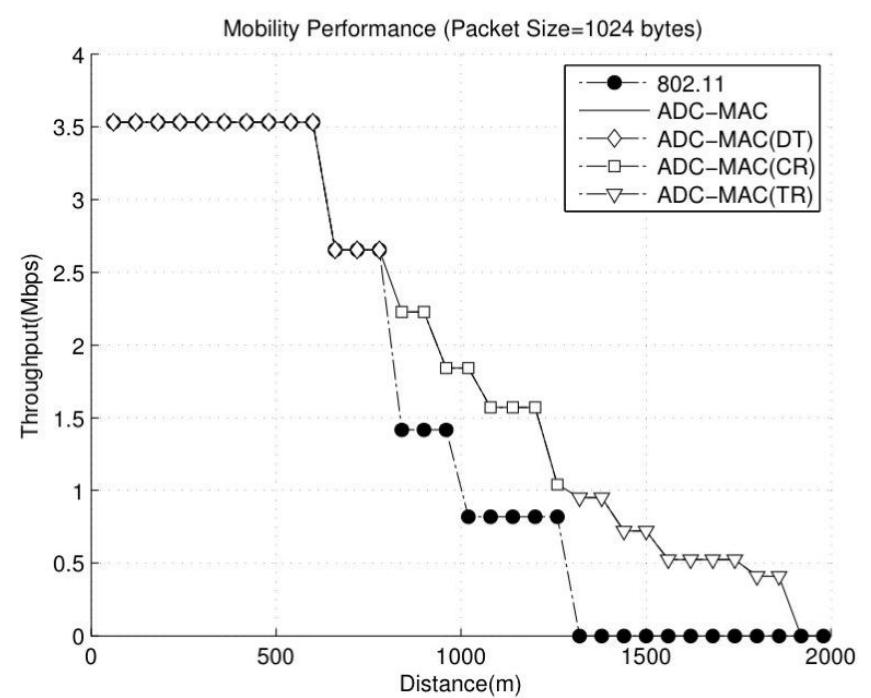

Fig. 14. Mobility performance comparison between DC-MAC protocol and 802.11 protocol

possible without the excellent support from Union Pacific Railroad, BNSF Railway, and Association of American Railroads (AAR).

\section{REFERENCES}

[1] J. J. Blum, A. Eskandarian, and L. J. Hoffman, "Challenges of intervehicle ad hoc networks," IEEE Transactions on Intelligent Transportation Systems, vol. 5, no. 4, pp. 347-351, 2004.

[2] B. Parno and A. Perrig, "Challenges in securing vehicular networks," Proceedings of the Fourth Workshop on Hot Topics in Networks (HotNets-IV), vol. 6, no. 4, pp. 347-351, November 14-15 2005.

[3] ANSI-IEEE 802.11 Standard:, "Wireless lan medium access control (MAC) and physical layer (PHY) specifications 802.11," 1999.

[4] M. V. Clark, K. K. Leung, B. McNair, and Z. Kostic, "Outdoor IEEE 802.11 cellular networks: Radio link performance," IEEE International Conference on Communications (ICC), 2002.

[5] S. Olariu and M. C. Weigle, Vehicular Networks: From Theory to Practice. CRC Press, 2009.

[6] M. Heusse, F. Rousseau, G. Berger-Sabbatel, and A. Duda, "Performance anomaly of 802.11 b," IEEE International Conference on Computer Communications (INFOCOM), 2003.

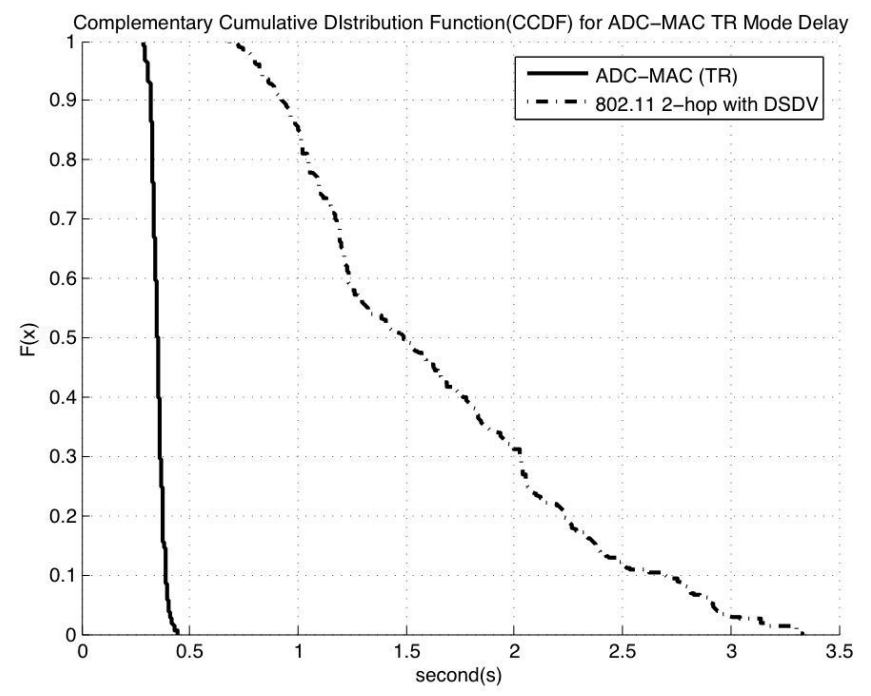

Fig. 15. Two-hop delay performance comparison between TR mode of DCMAC protocol and 802.11 protocol with DSDV routing protocol

[7] H. Zhu and G. Cao, "rDCF: A relay-enabled medium access control protocol for wireless ad hoc networks," IEEE Transactions on Mobile Computing, vol. 5, pp. 1201-1213, 2006.

[8] P. Liu, Z. Tao, and S. S. Panwar, "A cooperative mac protocol for wireless local area networks," IEEE International Conference on Communications (ICC), 2005.

[9] P. Liu, Z. Tao, S. Narayanan, and T. Korakis, "CoopMAC: A cooperative MAC for wireless LANs," IEEE Journal on Selected Areas in Communications (JSAC), vol. 25, no. 2, pp. 340-354, February 2007.

[10] M. Gokturk and O. Gurbuz, "Cooperation in wireless sensor networks: design and performance analysis of a MAC protocol," IEEE International Conference on Communications (ICC), pp. 4284-4289, May 2008.

[11] M. Gokturk and O. Gurbuz, "Cooperative MAC protocol with distributed relay actuation," IEEE Wireless Communications and Networking Conference (WCNC), 2009.

[12] H. Zhao, J. Wei, and Y. Xi, "A rate-adaptive cooperative MAC protocol based on RTS/CTS scheme for MANETs," ACCESSNETS 2007, pp. 1-6, August 2007.

[13] J. Zhang, Q. Zhang, and W. Jia, "VC-MAC: A cooperative MAC protocol in vehicular networks," IEEE Transactions on Vehicular Technology, vol. 58, no. 3, pp. 1-6, 2009.

[14] S. Sayed, Y. Yang, and H. Hu, "Throughput analysis of cooperative access protocol for multi-rate WLANs," IEEE Wireless 
Communications and Networking Conference (WCNC), pp. 1-6, April 2009.

[15] G. Bianchi, "Performance analysis of the IEEE 802.11 distributed coordination function," IEEE Journal on Selected Areas in Communications (JSAC), vol. 18, no. 3, pp. 535-547, 2000.

[16] H. Wu, Y. Peng, K. Long, S. Cheng, and J. Ma, "Performance of reliable transport protocol over IEEE 802.11 wireless LAN: Analysis and enhancement," IEEE International Conference on Computer Communications (INFOCOM), pp. 599-607, 2002.

[17] S. Ci, H. R. Sharif, and P. Mahasukhon, "Evaluating saturation throughput performance of the IEEE 802.11 MAC under fading channels," IEEE BroadNets, pp. 676-681, 2005.

[18] C. H. Foh, M. Zukerman, and J. W. Tantra, "A Markovian framework for performance evaluation of IEEE 802.11," IEEE Transactions on Wireless Communications, vol. 6, pp. 12761265, 2007.

[19] http://www.atheros.com/pt/bulletins/AR5002X.pdf.

[20] NS-2, "Network simulator," http://www.isi.edu/nsnam/ns.

[21] R. J.Punnoose, P. V.Nikitin, and D. D.Stancil, "Efficient simulation of ricean fading within a packet simulator," IEEE Vehicular Technology Conference (VTC), vol. 2, pp. 764-767, September 2000.

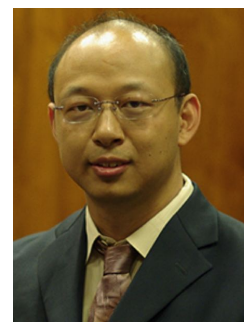

Ting Zhou received the B.S. degree in electrical engineering from Xidian University, China in 1996, the M.S. degree in Institute of Acoustics, Chinese Academy of Sciences, China, in 2001, and the Ph.D. degree in computer engineering from the University of Nebraska-Lincoln, USA, 2010. He is currently a research assistant at the Telecommunications Engineering Laboratory (TEL), Peter Kiewit Institute, University of Nebraska-Lincoln. His research interests include mobile cooperative wireless communications, embedded system designs and QoS aware

scheduling algorithms.

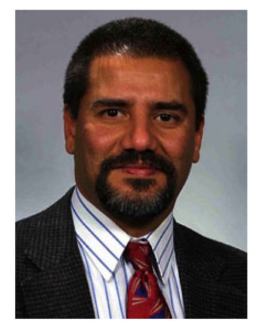

Hamid Sharif is the Henson Distinguished Professor of the Computer and Electronics Engineering Department and the Director of Advanced Telecommunications Engineering Laboratory (TEL) at the University of Nebraska-Lincoln. His current research interests include protocols, security, QoS and performance evaluations in wireless communication. He has authored or co-authored over 160 technical papers in major international journals and conferences. He has been serving as Steering Committee member, Symposium Chair and General Co-Chair of many international conferences. He is also contributing as the Editor, Associate Editor, or Editorial Board member for several journals. He is the Co-Editor-in-Chief of Wiley's Security and Communication Networks. He is a senior member of many professional organizations including IEEE. He is the current Chapters Coordinator for the IEEE Region 4 and the past Chair of the IEEE Nebraska Section and past President of the IEEE Nebraska Computer and Communications Societies.

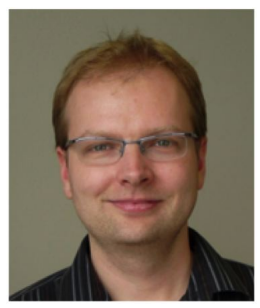

Michael Hempel received his Ph.D. in computer engineering at the University of Nebraska-Lincoln, USA. He is currently working as a Research Assistant Professor at the Advanced Telecommunication Engineering Laboratory (TEL) at the University of Nebraska-Lincoln. His research interests include wireless communication protocol design and performance analysis, wireless multimedia services, and distributed computing. For his research in networking he has also been developing various network simulation and analysis tools for streaming media and WiFi/WiMAX technologies. He has authored or co-authored over 50 publications in major international journals and conferences, and served as a TPC member on numerous international conferences.

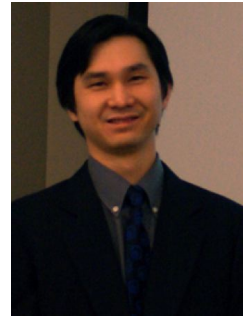

Puttipong Mahasukhon received the B.Eng. degree in electrical engineering from Kasetsart University, Bangkok, Thailand in 2000, the M.S. degree in telecommunications engineering from University of Nebraska-Lincoln, USA, in 2004, and the Ph.D. degree in computer engineering from the University of Nebraska-Lincoln, USA, 2010. In 2001, he was with the Communication Solution Co., Ltd. (TCS) in Thailand as a network engineer. He is currently a research assistant at the Telecommunications Engineering Laboratory (TEL), Peter Kiewit Institute, University of Nebraska-Lincoln. His research interests are in the areas of performance modeling, analysis, and optimization for Wireless LANs, Wireless Broadbands, and Wireless Sensor Networks.

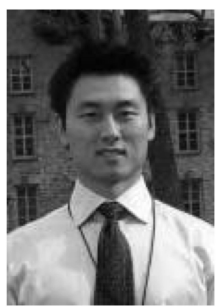

Wei Wang (M'10) received his BS and MS degrees from Xian Jiaotong University, China, in 2002 and 2005 respectively, and Ph.D. degree from University of Nebraska - Lincoln, USA, in 2009. He is currently an Assistant Professor at department of Electrical Engineering and Computer Science, South Dakota State University, USA. He is one of the recipients of the best paper award in IEEE WCNC 2008. He also served as a TPC member in IEEE Globecom 2010, WiMob 2008, 2009 and IEEE WCNC 2009, as well as a reviewer for several journals/transactions. His research interests include computer networks, wireless communications, embedded systems and multimedia computing.

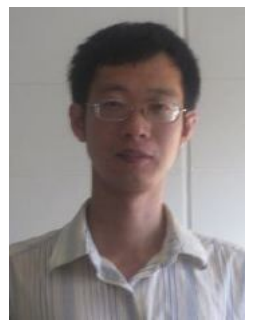

Tao Ma received the B.Sc. and M.Sc. degrees from Xian Jiaotong University, China, in 2002 and 2005 respectively, both in electrical engineering. He is currently pursuing the Ph.D. degree and in Department of Computer and Electronics Engineering, University of Nebraska-Lincoln. His research areas are Cross-layer design for QoS provisioning in wireless data networks, and multi-media distribution. 